Linguistique, littérature, didactique

$157-158 \mid 2013$

Théories et pratiques des genres

\title{
Types, genres et généricité en débat avec Jean- Michel Adam
}

Driss Ablali

\section{(2) OpenEdition}

Journals

\section{Édition électronique}

URL : http://journals.openedition.org/pratiques/3850

DOI : 10.4000 /pratiques.3850

ISSN : 2425-2042

\section{Éditeur}

Centre de recherche sur les médiations (CREM)

\section{Édition imprimée}

Date de publication : 1 juin 2013

Pagination : 216-232

\section{Référence électronique}

Driss Ablali, « Types, genres et généricité en débat avec Jean-Michel Adam », Pratiques [En ligne], 157-158 | 2013, mis en ligne le 18 décembre 2017, consulté le 07 mai 2019. URL : http:// journals.openedition.org/pratiques/3850; DOI : 10.4000/pratiques.3850

(c) Tous droits réservés 


\title{
Types, genres et généricité en débat avec Jean-Michel Adam
}

\author{
Driss Ablali \\ Praxitexte/CREM - Université de Lorraine
}

\begin{abstract}
"Une science ne commence d'exister ou ne peut s'imposer que dans la mesure où elle fait exister et elle impose ses concepts dans leur dénomination »

E. Benveniste
\end{abstract}

Dans la perspective des recherches textualistes qui tentent de problématiser les enjeux linguistiques des typologies textuelles, nous souhaitons, dans ce qui suit, contribuer à la réflexion portant sur les fondements épistémologique et théorique des notions de " type », de "genre » et "généricité », telles qu'elles ont été théorisées en linguistique textuelle par J.-M. Adam. Plusieurs questions se posent et méritent qu'on s'y attarde. La première concerne le(s) cadre(s) théorique(s) dans le(s)quel(s) ces trois notions sont définies. La deuxième porte sur la transversalité des propositions élaborées par Adam et Heidmann pour l'étude de la généricité. Pour finir, nous mettrons l'accent sur la question des observables pour montrer qu'une caractérisation multi-sémiotique des genres ne peut se faire que sur corpus.

\section{Préambules}

La bibliographie des travaux de J.-M. Adam depuis près de trente ans prouve, à l'évidence, son intérêt permanent pour la question du texte. Ses travaux, qui font autorité en la matière, sont au fondement de la linguistique textuelle. En effet, comme en témoignent ses publications des années soixante-dix ${ }^{(1)}$, Adam fait partie des linguistes qui ont permis que la linguistique s'émancipe du cadre phrastique dans lequel elle a longtemps été cantonnée. De ses premières publications, ancrées dans le champ de la sémiotique, à ses derniers écrits, traitant de la textualité du discours littéraire ${ }^{(2)}$, la place accordée aux genres a été constante mais de façon évolutive. Nous ne cherchons pas ici à faire une présentation chronologique détaillée de la pensée textualiste de ce linguiste et essaierons simplement d'opérer

(1) Cf. Adam (1975), (1976), (1979).

(2) Heidmann \& Adam (2010) est notre terminus ad quem dans cette étude. 
une lecture visant à mettre en perspective ses positions théoriques et épistémologiques afin de mieux comprendre les rapports d'interdépendance qu'entretiennent dans ses recherches les catégories de textes, genres et discours.

Si nous nous intéressons aux travaux d'Adam, ce n'est pas avec une visée polémique contrairement à certaines habitudes du débat académique. Au contraire, nous avouons avoir ressenti un réel plaisir intellectuel à suivre sur plus de trente ans l'évolution des enjeux heuristiques de son approche du texte, avec une attention particulière pour les trois catégories descriptives mentionnées dans le titre de cette contribution. Ce qui ne nous empêchera pas, car nous croyons au débat scientifique, de tenter d'évaluer la pertinence et la cohérence des propos tenus sur la place et le rôle des typologies textuelles dans l'analyse des discours et des corpus. Précisons également que dans l'article qui suit ce n'est pas l'ensemble des travaux d'Adam, tels qu'ils portent sur le style, l'argumentation ou l'analyse des discours de la presse, que nous cherchons à étudier. Notre projet, on l'a dit, est bien plus modeste et se limite à repérer le lien entre trois catégories descriptives : «type », " genre » et « généricité », et leur socle épistémologique. Nous ne nous interdirons pas, pour autant, de faire allusion, chaque fois que cela paraîtra nécessaire, à d'autres catégories descriptives pour appuyer certains arguments ou pour compléter les catégories en question.

Pour clore ce préambule, nous dirons que l'essentiel de notre débat avec Adam portera sur les questions suivantes : quelles sont les bases épistémologiques qui ont servi de référence pour la teneur des typologies textuelles effectivement proposées par l'auteur? Y a-t-il un noyau épistémologique et méthodologique commun à l'ensemble de ses réflexions sur les catégories de type, genre et généricité ? Le passage de type à genre, et de genre à généricité, comme il est revendiqué plusieurs fois dans ses travaux des dix dernières années, est-il synonyme de coupure épistémologique ou correspond-il à une simple évolution terminologique?

\section{Des séquences textuelles aux genres : quelle(s) épistémologie(s) ?}

Derrière les notions et les concepts se profilent des questions qui sont loin d'être aussi mineures qu'on puisse le penser. Des premières publications relevant de la grammaire de texte aux derniers travaux «préconisa(n)t l'intégration de la narratologie dans la linguistique textuelle et celle de la théorie du texte dans l'analyse de discours ", (2010:3), il est très difficile de trouver dans la réflexion d'Adam sur les typologies, comme on le verra plus bas, une stabilité épistémologique.

Sa conception du texte et du discours n'a pas cessé d'évoluer mettant à l'épreuve toute tentative de cerner son socle épistémologique et méthodologique. Il est d'ailleurs nécessaire de souligner que lorsqu' on parcourt l'ensemble de la réflexion de l'auteur, depuis les années soixante-dix jusqu'à ses dernières publications sur le genre du conte, on constate une absence de cohérence au niveau des postures théoriques. Sur ce point il faut suivre 1'histoire : de la sémiotique à la grammaire générative et la psychologie cognitive, en passant par la pragmatique, la narratologie et la poétique, l'empan conceptuel développé par Adam sur la base de toutes ces disciplines pour l'analyse du texte est trop large pour fonder un ensemble structuré et articulé de concepts invariables, canoniquement fixés. La simple énumération des champs disciplinaires que nous venons de faire, de notre point de vue pose problème dans la mesure où nous estimons que l'efficacité des concepts et des notions, bref d'une théorie quelconque, dépend d'une armature bien charpentée, soumise à l'invariance des postulats. C'est l'intérêt d'une théo- 
rie du texte de chercher à travers ses concepts et ses principes leurs points communs et leurs interrelations pour dégager au travers de leurs applications, ce qui les unit tous. Certes 1'idée d'une dynamique évolutive dans la théorisation est nécessaire pour parvenir jusqu'aux principes généraux constants et réguliers, mais à condition que l'échafaudage de la théorie reste intact. Or chez Adam les bases d'une théorie du texte sont multiples, changeantes et en permanent devenir, comme le sont également ses catégories descriptives mises en œuvre pour l'analyse des textes.

Pour en finir, ou sortir, si l'on veut, de ces questions théoriques, faisons une mise au point historique, qui s'ouvre elle-même sur une constatation apparemment inattendue : quand on lit les textes des années soixante-dix, on ne peut manquer de repérer chez Adam l'influence de la sémiotique, du matérialisme historique et de la grammaire générative sur le cadre théorique de ses analyses des textes publicitaires, des poèmes et des textes littéraires. Sans entrer dans un vétilleux décompte du nombre des pages réservé à telle ou telle discipline, il est au premier coup d'œil évident que des sémioticiens y occupent une place de premier ordre comme l'attestent les considérations sur le "sémiotique » et le «symbolique » (Kristeva), sur la « logique narrative » (Brémond), sur la «cohésion sémantique » inspirée de l' « isotopie »(Greimas). Ces considérations épistémologiques ne peuvent être passées sous silence, car elles conditionnent le cadre conceptuel et la démarche à suivre, voire les critères de validité de l'analyse. Deux citations suffisent ici pour illustrer cette influence, toutes les deux tirées des travaux de l'auteur publiés dans les années soixante-dix : "Comme je m'appuie ici sur des travaux de Julia Kristeva dont il n'a guère été fait état dans Pratiques, il faut commencer par brièvement situer mon propos ». (1979: 105. C'est nous qui soulignons).

Dans une autre analyse portant sur le texte publicitaire, datant de la même décennie, c'est le matérialisme historique d'Althusser et Pêcheux, comme en témoigne ce passage, sur lequel reposent les présupposés méthodologiques et épistémologiques pour étudier le discours publicitaire : "Nous ne parlons plus, dès lors, de "message", mais de "discours" publicitaire, entendant par là la nécessité de lier linguistique, marxisme et psychanalyse, la nécessité de diffuser un savoir et non l'idéologie culturelle de la classe dominante. [...]. L'enjeu d'un travail sur la publicité n'est donc rien moins qu'une interrogation sur la fonction idéologique du discours, sur les conditions idéologiques de reproduction des rapports de production ». (1975:62. C'est nous qui soulignons).

Ce déséquilibre est à notre sens révélateur. On est surpris de constater qu'Adam dispose parallèlement aux travaux d'inspiration marxiste d'une nouvelle heuristique pour analyser les textes venant cette fois-ci d'un courant qui s'est développé à l'opposé de la vulgate marxiste, en s'appuyant sur les avancées théoriques et épistémologiques de Hjelmslev, Propp et Merleau-Ponty, à savoir la sémiotique narrative. C'est du moins ce qui ressort, à nos yeux, de la citation suivante : « A ce niveau de la recherche, il nous semble nécessaire que la grammaire de texte intègre une part de la grammaire du récit. [...] Les recherches de A.J. Greimas, T. Todorov et surtout $C l$. Bremond peuvent être reconsidérées ici $[. .$.$] . Le projet de \mathrm{Cl}$. Bremond a l'avantage de nous permettre d'intégrer la grammaire de texte dans le discours littéraire et plus particulièrement romanesque » (1976:213-214. C'est nous qui soulignons). Mais quelques pages plus haut, c'est le générativisme qui sert de toile de fond épistémologique à la partie empirique, comme en témoigne cette citation : «Les limites du structuralisme sont évidentes. En tant que théorie descriptive, il ne peut rendre compte des conditions de production des discours. [...] D’où la né- 
cessité de s'intéresser au développement des grammaires génératives et de considérer les perspectives qu'elles ouvrent pour l'analyse du discours littéraire » (1976 : 195. C'est nous qui soulignons).

On voit à quel point les soubassements épistémologiques d'Adam ne sont pas faciles à cerner notamment sous l'effet de l'hétérogénéité des paradigmes au sein desquels il inscrit simultanément ses travaux. Le paradigme sémiotique de Greimas ${ }^{(3)}$ et Brémond n'a rien à voir avec le générativisme de Chomsky, encore moins avec l'analyse de discours à la "française », laquelle a fait le choix de suivre non pas l'auteur de Structures syntaxiques, mais le distributionnalisme d'Harris. Ces théories renvoient à des convictions épistémologiques antagonistes qui n'ont ni la même sensibilité, ni la même histoire.

L'absence de cadres théoriques et épistémologiques homogènes se manifeste aussi par l'intégration ultérieure de nouvelles heuristiques qui mettent en cause celles élaborées dans les années soixante-dix.

En effet, cette inscription des travaux d'Adam entre analyse de discours, générativisme et sémiotique, ne va pas perdurer. Dans les années quatre-vingts ${ }^{(4)}$, ces positions vont faire place à de nouveaux paradigmes théoriques issus des Textlinguistik, des recherches cognitives et de la pragmatique. Au sujet des allemands, notamment ceux de Weinrich, J. Petöfi F. Danes, H. Rück, K. Hatakeyama, E. Söser, Adam lui-même s'en explique dans les termes suivants : "Ces traductions ont accéléré ma prise de conscience des limites de la sémiotique de l'école de Paris et de l'analyse du discours française ». (2010:33). Position confirmée dans les premières pages des Éléments de linguistique textuelle : «Pour conclure cette section sur la structuration séquentielle des textes, il faut bien localiser l'originalité des propositions du présent ouvrage en l'opposant à deux grandes tentatives : celle de la sémiotique narrative d'A. J. Greimas et de l'École de Paris, d'une part, celle de l'analyse conversationnelle d'E. Roulet, d'autre part » (1990: 94). En ce point, il est remarquable de voir une fois de plus se conjoindre des heuristiques inconciliables pour fonder une nouvelle discipline qu'Adam appelle « la linguistique pragmatique et textuelle » ${ }^{(5)}$, et ce sont les cognitivistes qui viendront à la rescousse parallèlement aux influences de Textlinguistik, . On remarquera tout spécialement la théorie psycho-cognitive des schémas, développée surtout par Kintsch et Van $\operatorname{Dijk}^{(6)}$ qui sert de soubassement à la question des séquences. L'attestent de façon incontestable des formules telles que : «Mes propres travaux doivent beaucoup aux thèses de celui qui collaborera très tôt avec le psychocognitivisme Walter Kintsch. Ma théorie des séquences et la réflexion présente sur les genres sont issues d'une discussion des "superstructures textuelles" proposées par T. A. Van Dijk et de la théorie cognitive des schémas » $(1999: 10)^{(7)}$. Dans le même ouvrage,

(3) Au sujet des rapports entre sémiotique et linguistique, on pourra lire Ablali (2013 b).

(4) Ici il faut souligner que c'est dans la thèse de V. Portillo Serrano, Problématique des genres dans les productions écrites universitaires : cas du résumé scolaire chez des étudiants français et mexicains, soutenue à l'Université de Franche-Comté en 2010, que nous avons rencontré plusieurs citations tirées des travaux d'Adam qui nous ont permis de mieux appréhender les questions théoriques et épistémologiques traitées dans cette contribution. Nous les signalerons le moment venu.

(5) «La linguistique pragmatique et textuelle que le présent ouvrage tente de situer, se définit par une attention non seulement à la nature des parties, mais également aux différentes manifestations d'un “tout" textuel» (1991:17).

(6) Cf. Portillo Serrano (2010:17-22).

(7) Cité par Portillo Serrano (2010:38). 
les dettes se multiplient jusqu'à la psychologie cognitive et la psycholinguistique textuelle : «Ayant, dans un cadre transdisciplinaire, suivi de très près les travaux en particulier des laboratoires de Dijon (Fayol) et de Poitiers (Esperet, Gaonac'h, Passerault), je tiens à souligner ici une dette épistémologique qui transparaît clairement dans mes éléments de linguistique textuelle (1990)» (1999: 15). Ou encore à l'égard de Meschonnic : «Comme je l'ai déjà dit dans le Style dans la langue, mon analyse de ce contexte éditorial rejoint épistémologiquement celle que formulait Henri Meschonnic... » (1999 : 19). Dans cette discipline, « la linguistique pragmatique et textuelle », convergent des mouvements aux présupposés extrêmement hétérogènes. Bref, c'est un trait caractéristique d'Adam, le fait qu'il change de modèle en permanence si bien que chaque décennie remet en cause le fondement théorique et épistémologique de la décennie précédente. Ce que les années 2000 ne vont pas démentir, et de façon répétitive, notamment dans le passage suivant, qui précise que la visée principale des travaux d'Adam est de « sortir définitivement la linguistique textuelle du logicisme formaliste de la "grammaire de texte" et la définir comme une discipline du champ de l'analyse des discours. » (Adam $2003: 51$ ), d'où une nouvelle appellation pour désigner ses recherches, que l'auteur appelle « analyse textuelle du discours ». Ce nouveau projet revendique des dettes envers, entre autres, E. Benveniste, M. Bakhtine et en particulier envers E. Coseriu. Peut-être sommes-nous ici devant la phase la plus aboutie du projet textuel d'Adam, avec cet ancrage linguistique, loin des postulats universalistes du cognitivisme et du formalisme de la pragmatique, mais seul l'avenir pourra nous le confirmer.

\section{Des types aux genres}

Une fois effectuée cette reconstitution épistémologique, il reste à nous interroger sur la question des catégories descriptives, et, corrélativement, sur leur rapport aux heuristiques revendiquées par Adam pour l'analyse des textes. C'est en ce point qu'intervient, de façon déterminante, une autre évolution, parallèle à celle des positions théoriques qu'on vient d'évoquer, celle des pratiques. Une seule catégorie retiendra notre attention à ce sujet, celle des «types de texte». Adam propose dans les années quatre-vingt sept types textuels : «récit, description, argumentation, exposition, injonction-instruction, conversation et poème », auxquels il renoncera quelques années plus tard pour développer une typologie séquentielle, afin de rendre compte de l'hétérogénéité des textes ${ }^{(8)}$ (1992). Les modifications de ce type sont fortement redondantes dans les écrits des années quatre-vingt-dix. Voici la plus pertinente : «Pour une typologie de type (e), il existe de grands types de textes. Je citerai, sans justifier ici la limitation de l'énumération : le récit, la description, l'argumentation, l'exposition (avec ses sous-types explicatif et compte rendu d'expérience), l'injonction-instruction, la conversation, le "poème" (ou type autotéliquepoétique). A ce niveau de généralité - que j'ai adopté dans mes travaux antérieurs -, nous avons affaire à une typologie trop globale qui ne tient pas assez compte de l'hétérogénéité des textes eux-mêmes. » (1987:55).

Adam propose de substituer aux typologies textuelles, une typologie basée sur la séquentialité. Son point nodal : «Les textes sont des structures tellement diverses et complexes qu'il est impossible d'en établir une typologie sauf par commodités pédagogiques illusoires. En revanche, on peut repérer des segments de plus petite taille, généralement composés de plusieurs phrases : les séquences. »

(8) Cf. Adam (1992). 
(1991 : 9). Deux choses sont à signaler dans cette citation. La première : les régularités des textes n'existent pas, la deuxième, conséquence directe de la précédente : la séquence est l'unité constituante du texte, seule capable de rendre compte de son hétérogénéité. Il convient, avant de poursuivre, de rappeler que cet intérêt pour les typologies est parallèle, au niveau théorique, aux grandes influences des modèles cognitifs, évoqués il y a quelques instants. En témoigne aussi l'esprit universaliste qui accompagne l'analyse des textes en termes de séquences, débouchant sur une conception qui écarte la différence, transposable à tout type de textes, d'où la notion de "prototype » pour définir les types de séquences.

Autre aspect du problème, que nous ne ferons également que signaler : les variations de l'attitude d'Adam à l'égard des catégories descriptives qui jouent sur un binarisme redondant : chaque concept-fétiche a son cadre théorique. La catégorie «types de textes » est solidaire de la grammaire de texte, celle de la séquentialité de la pragmatique textuelle, celle des genres de la linguistique textuelle, et pour terminer celle de la généricité de l'analyse textuelle des discours. Il n'y a donc pas que les orientations théoriques et épistémologiques qui changent, mais les catégories aussi. C'est ce qui pourrait peut-être expliquer pourquoi les réflexions d'Adam n'ont jamais pu s'ériger en théorie qui cherche à rassembler, ordonner et unir ses concepts, et les organiser autour d'un petit nombre d'idées-forces comme la marque d'une heuristique qui unit les catégories descriptives à un cadre théorique solide et inflexible. Ce manque d'autonomie épistémologique, qui oblige à revenir sans cesse sur la plausibilité des catégories, des hypothèses et des cheminements qui ont conduit à l'élaboration des schémas et des modèles, contrarie la cohérence de son projet scientifique, et pourrait marquer ses limites, ou restreindre sa portée. Car si un tel socle épistémologique n'existe pas, alors c'est le projet même d'une caractérisation des genres textuels qui pose problème. Qu'est-ce donc, en effet, qu'une théorie, sinon l'articulation d'une pratique ? Et qu'est-ce qu'une épistémologie, sinon le discours qui élucide ce rapport?

\section{Six propositions pour la "généricité "}

Pour aborder l'ultime catégorie descriptive proposée par Adam, dans ses derniers travaux, celle de "généricité », soulignons de prime abord que son apparition est la suite logique de la décontextualisation et de la dissociation entre « texte » et « discours » que préconisaient les Éléments de linguistique textuelle. En témoigne cette citation, qui montre que les travaux d'Adam connaissent aujourd'hui une nouvelle phase en situant résolument la linguistique textuelle dans le domaine plus vaste de l'analyse du discours :

«Pour aborder le concept de contexte, je dois repartir de la malheureuse reprise (Adam 1990:23 \& 1999 : 39) de la formule Discours = Texte + Contexte/conditions de production et de réception-interprétation et de son symétrique Texte $=$ Discours - Contexte/conditions de production. Cette formule, en hésitant entre contexte et conditions de production du discours, a manifestement son origine dans l'Analyse de Discours française des années 1960-80. Je dirai donc qu'il faut aujourd'hui l'écarter pour deux raisons. D'abord parce qu'elle laisse entendre une opposition et une complémentarité des concepts de texte et de discours alors qu'il s'agit de dire que ces deux concepts se chevauchent et se recoupent avant tout en fonction de la perspective d'analyse choisie. Ensuite parce qu'il faut tenir compte de ce que Jacques Guilhaumou (1993 \& 2002 : 32) décrit comme le passage d'une conception sociolinguistique de l'AD à sa redéfinition comme « discipline herméneutique à part entière ». (2006 b). 
Du point de vue de la relation texte/genre/discours chez Adam, les typologies textuelles ne sont pas aussi stables que chez d'autres spécialistes de ces questions. Au lieu de parler du sens d'un texte en relation avec un genre, l'auteur insiste sur le fait que rien n'empêche un seul texte de développer des configurations linguistiques renvoyant à plusieurs catégories génériques, d'où le choix, pour rendre compte de cet aspect multi-générique et dynamique, d'opter plus pour la notion de " généricité » que pour celle de " genre » :

«La généricité est, en revanche, écrit Adam, la mise en relation d'un texte avec des catégories génériques ouvertes. Cette mise en relation repose sur la production et/ou la reconnaissance d'effets de généricité, inséparables de l'effet de textualité. Dès qu'il y a texte — c'est-à-dire la reconnaissance du fait qu'une suite d'énoncés forme un tout de communication -, il y a effet de généricité - c'est-à-dire inscription de cette suite d'énoncés dans une classe de discours. La généricité est une nécessité socio-cognitive qui relie tout texte à l'interdiscours d'une formation sociale. Un texte n'appartient pas, en soi, à un genre, mais il est mis, à la production comme à la réception-interprétation, en relation à un ou plusieurs genres» (2004: 62).

Pour revenir à la question de la généricité qui nous intéresse ici, Adam rappelle que le renoncement au concept de genre, jugé « trop statique », au profit de celui de généricité permet de passer d'une conception taxinomique des genres comme «répertoire » vers une conception « dynamique », postulant que le sens du texte à travers la diversité des genres n'est pas cantonné en un lieu représenté par une simple étiquette, mais qu'il circule, sémiotiquement, de manière transversale entre les instances « énonciative», " éditoriale » et « lectoriale ». C'est le genre lui-même, en tant que système, qui se voit résolument mobilisé et impliqué dans les mouvements et les tensions engendrés par la dynamique intergénérique. Nous citons un passage parmi les plus explicites:

«Ce que nous définissons comme la généricité d'un texte résulte d'un dialogue continu, souvent conflictuel, entre les instances énonciative, éditoriale et lectoriale. Un texte relevant généralement de plusieurs genres, il ne s'agit plus de le classer dans une catégorie - son appartenance -, mais d'observer les potentialités génériques qui le traversent - sa participation à un ou plusieurs genres - en tenant compte des points de vue tant auctorial qu'éditorial et lectorial. Analyser une participation au lieu de se limiter à une appartenance classificatoire permet d'entrer dans la complexité des faits de discours. À l'exclusion de genres socialement très contraints, la plupart des textes ne se conforment pas à un seul genre, mais opèrent un travail de transformation d'un genre à partir de plusieurs genres (plus ou moins proches)» (Adam \& Heidmann $2007: 27$ ).

En ce point, il est remarquable de voir une fois de plus se conjoindre catégories descriptives et cadre théorique : car le passage de la notion de genre à celle de généricité représente, pour les auteurs, un changement de paradigme dans la mesure où les six propositions dont il sera question ci-après permettraient de traiter de la « dynamique socio-cognitive » des genres. Tout cela dans un nouveau paradigme que les auteurs appellent maintenant «l'Analyse textuelle des discours », que l'on pourrait appeler « analyse textuelle du discours littéraire ${ }^{(9)} »$, car toutes les propo-

(9) Ici on pourra rappeler que ces six propositions sont placées dans l'introduction du Texte Littéraire. Pour une approche interdisciplinaire, emplacement qui sert de fondation à l'analyse empirique de tous les discours littéraires convoqués dans cet opus. Adam a travaillé sur plusieurs types de discours, personne ne peut le contester : le discours politique, journalistique, publicitaire, procédural, etc. Mais sa réflexion depuis les années 2000 a fait le choix de se placer essentiellement dans la perspective du discours littéraire. 
sitions qu'Adam \& Heidmann (dorénavant A\&H) ont développées dans les dix dernières années, l'ont été essentiellement sur un seul discours, le discours littéraire. Il nous reste à déterminer si les six propositions qui sont au centre de la notion de généricité et qui ne manquent pas de poser de sérieux problèmes théoriques et méthodologiques, peuvent être rapportées à tous les genres, comme le veulent les deux auteurs, ou si elles sont spécialement fondées sur des canons littéraires qui neutralisent leur possible généralisation. La question que l'on est en droit de se poser, en examinant ces propositions sur la généricité, est bien celle de leur valeur ajoutée. Que peut apporter en effet une nouvelle réflexion sur la catégorie du genre, regroupée en six propositions, aux typologies textuelles?

\section{1. « Tout texte participe d'un ou de plusieurs genres"}

Cette première proposition qu'A\&H développent à partir de la réflexion de J. Derrida ne convient pas au linguiste, car elle est spécialement fondée sur des postulats philosophiques qui n'ont rien de linguistique. Rappelons d'abord les raisons avancées par le philosophe pour affirmer que le texte est une expérience singulière, qui participe de plusieurs genres, et reste foncièrement inclassable. Dans un texte intitulé « La loi du genre », Derrida procède à l'analyse d'un texte de M. Blanchot, texte qui mine les catégories esthétiques traditionnelles. Ici il convient de noter que Blanchot est d'abord une figure du siècle dernier qui a toujours cherché à dénier la validité de la notion de genre ${ }^{(10)}$, comme le fera R. Barthes, après lui. Du point de vue de Blanchot, le rôle de la littérature consiste justement à déconsidérer les normes génériques en faveur de l'œuvre dans son unicité. Il est d'ailleurs arrivé à Blanchot de modifier la mention de genre, d'une version à l'autre ou d'une édition à l'autre de ses écrits ; c'est notamment, comme le rappelle Derrida, le cas de L'arrêt de mort, où la mention « récit » est effacée d'une version à l'autre, ou encore de La folie du jour, texte édité sans aucune mention de genre, « récit» ou « roman », contrairement aux deux dernières pages de ce texte, qui évoquent à quatre reprises le mot « récit» " pour nommer le thème, le sens ou l'histoire, le contenu ou une partie du contenu de La folie du jour » (1986 : 267). En partant d'un seul auteur et à partir de cette expérience, Derrida en arrive à la conclusion qu' " un texte ne saurait appartenir à aucun genre. Tout texte participe d'un ou de plusieurs genres, il n'y a pas de texte sans genre, il y a toujours du genre et des genres mais cette participation n'est jamais une appartenance. Et cela non pas à cause d'un débordement de richesse ou de productivité libre, anarchique et inclassable, mais à cause du trait de participation luimême, de l'effet de code et de la marque générique. Si la remarque d'appartenance appartient sans appartenir, participe sans appartenir, la mention de genre ne fait pas simplement partie du corpus » (Derrida 1986 : 264) (souligné par l'auteur).

Il y a lieu sans doute de se demander si cette proposition peut être rapportée à tous les textes. Certainement pas, si l'on pense simplement que les textes globalement n'ont pas le pouvoir de modifier leur genre, ils n'ont aucune autonomie par rapport à leur genre, celui dont ils relèvent. Car cette appartenance est le fondement même du texte. Le texte est intrinsèquement marqué par son genre. Le texte emprunte la voix que lui dicte son genre, lequel contraint un ton, une composition,

(10) Il en dit ceci : «Le genre est une pure convention dont il convient de se passer au nom du caractère "unique" de l'œuvre : "Seule importe l'œuvre, [...] le poème dans sa singularité resserrée [...]. Seul importe le livre, tel qu'il est, loin des genres, en dehors des rubriques [...], sous lesquelles il refuse de se ranger et auxquelles il dénie le pouvoir de lui fixer sa place et de déterminer sa forme » (Blanchot 1959: 272). 
une syntaxe, une ponctuation, etc. Et c'est dans ce cadre du primat des contraintes génériques que les textes du même genre finissent par se faire écho.

L'appartenance est donc dans la durée, et les frontières ne sont pas mouvantes. Le statut générique d'un texte est constant, un CV ne peut pas embrasser plusieurs genres, un article de revue ne peut se revendiquer de statuts génériques différents, un topo de randonnée ne peut être réfractaire aux classements. Ces textes ont une textualité qui ne laisse place à aucune indécision générique. Car le trait de généricité n'est pas dans le paratexte, mais dans la textualité même du texte. Écrire dans un genre donné, c'est aussi connaître les normes typographiques qui sont imposées, la longueur à ne pas dépasser, les modes d'énonciation autorisés, les pronoms personnels exigés, les signes de ponctuation à éviter, les modes verbaux à privilégier. Ce sont bien les genres qui transmettent ces cooccurrences morphosyntaxiques et les confirment. On cherche ici à montrer que le genre n'est pas dans la simple mention de genre, il s'inscrit dans la matérialité sémiotique du texte, il est ainsi dégagé de la configuration d'ensemble que les interactions morphologiques, syntaxiques, sémantiques, énonciatives, stylistiques forment dans le corps même du texte. La proposition d'A\&H, « Tout texte participe d'un ou de plusieurs genres », est loin des réalités effectives des autres discours, et nous voudrions la remplacer par celle qui préconise que les genres sont atemporels tant qu'ils durent : nous écrivons et nous lisons dans un seul et même genre. Ici ce n'est pas l'identification d'un genre en tant que tel qui nous intéresse, mais comment le genre conditionne tous les paliers du texte, aussi bien dans la production que dans l'interprétation. On ne peut pas faire du multi-générique un principe constitutif des textes, car la majorité des textes ne sont pas soumis à cette «participation sans appartenance » que définit Derrida à partir d'un seul cas, celui de Blanchot, et qu’A\&H généralisent sur la base du discours littéraire.

\section{2. «Les genres sont aussi divers que les pratiques discursives"}

On peut s'interroger sur l'apport heuristique à l'« étude » de la diversité des genres textuels de cette proposition d'A\&H, qui prend appui sur des travaux aussi divers que ceux de Todorov, Bakhtine, Bronckart, Zima. Pour le dire clairement, «Les genres sont aussi divers que les pratiques discursives », est un constat simplement stipulé mais non engendré selon des règles linguistiquement attestées. Ce postulat mériterait d'être théoriquement développé. Dire que les sons sont aussi divers que les langues n'apporte rien pour le phonéticien, pas plus que de dire que les langues sont aussi diverses que les cultures pour l'anthropologue. Cette proposition ne détermine pas ce que sont empiriquement les règles du jeu du genre, car l'étude d'un genre implique d'abord de définir la pratique dont il dépend. Qu'on en juge : «Un genre ne se comprend donc qu'à l'intérieur d'un ensemble de ressemblances et de différences entre genres et sous-genres définis par un groupe social à un moment donné de son histoire culturelle et discursive ». (2007:23). On n'a pas besoin des autres genres pour caractériser un genre quelconque : ce n'est pas en explorant le roman que nous saisissons la spécificité du fait divers; ce n'est pas en lisant une recette de cuisine que nous apprenons à lire un article scientifique. Tous les genres ne s'influencent pas les uns les autres. Or les genres relevant d'une même pratique discursive, eux, obéissent à un autre fonctionnement : un genre s'oppose à toute une série de genres qui, appartenant à la même activité langagière, sont incompatibles entre eux : le choix, par exemple, de traiter d'un observable linguistique dans un rapport de thèse neutralise les autres genres du 
même discours, comme le compte rendu dans une revue scientifique, ou le chapitre dans un livre collectif, ou encore l'article dans une revue à comité de lecture. Ici nous sommes dans le même espace de normes qui est celui du discours scientifique. Car un genre ne se comprend qu'à l'intérieur d'un ensemble de genres appartenant à la même pratique langagière, comme l'indiquent clairement les travaux de F. Rastier depuis la fin des années quatre-vingt. Nous y reviendrons : ce réseau de relations nécessaires entre des genres du même discours implique en même temps que les genres ne sont pas indifférents les uns aux autres, mais qu'ils entretiennent au contraire les uns avec les autres une toile de fond commune constituée par le « discours » dans le sens que lui donne F. Rastier. Dans la mesure donc où la question du genre dépend de celle de la pratique, on comprend que le discours constitue l'unité fondamentale - l' " atome » - du champ de la typologie des textes. Un article de critique dans le discours journalistique et un article de critique dans le discours scientifique ne sont pas similaires ${ }^{(11)}$, ils n'ont aucune ressemblance, ni morphosyntaxique ni énonciative, ni typographique. La textualité épistolaire d'une lettre dans le discours littéraire diffère dans tous ses paliers de celle de la lettre dans le discours diplomatique ${ }^{(12)}$. Car les deux ne sont pas issues de la même pratique, littéraire pour l'une, diplomatique pour l'autre. Ainsi se trouve marqué de la façon la plus forte l'impact du discours comme palier : le discours comme interaction complexe et hétérogène au regard des genres recensés commande des fonctionnements langagiers foncièrement spécifiques aux genres qui le constituent. Il n'y aurait donc tout simplement pas de normes génériques et d'écarts entre genres s'il n'y avait pas de pratiques langagières habituelles communes et surtout diverses.

\section{3. « Les genres sont des pratiques normées, cognitivement et socialement indispensables »}

D'entrée de jeu, il convient de reconnaître, dans le prolongement de cette proposition, que le genre est un espace de contraintes, l'expression d'une réalité socio-cognitive institutionnalisée. Cette proposition aboutit à concevoir les genres comme des réalisations d'une ou plusieurs opérations normalisées d'abord linguistiques puis socio-cognitives propres à la langue utilisée. C'est là un argument pour élargir la dimension linguistique des genres à l'étude des processus sociocognitifs. Pour soutenir le principe de l'irréductibilité de l'étude des genres à la description linguistique, A\&H mettent l'accent sur le fait que le genre garde les traces cognitives et sociales des opérations productrices. Le genre, comme phénomène fortement investi socialement, est ainsi intriqué avec le culturel et le cognitif. Mais la question est plutôt de savoir comment le produit d'une activité langagière au sein d'un genre peut être rattaché à la dimension socio-cognitive du langage. Ainsi, ce n'est pas un simple ancrage du genre au socio-cognitif qui détermine ces rapports, mais plutôt une forte isomorphie entre le linguistique et le socio-cognitif qui permet de penser le statut de ces normes plurielles et communicantes dans les textes, et dont les auteurs ne disent rien. A notre avis, la question

(11) Cf. Ablali (2005).

(12) Contrairement à Adam (1998) qui parle étrangement de « discours épistolaire » et de ses genres, nous soutenons qu'il n'y a pas de discours épistolaire, car l'épistolaire ne peut être qu'un genre à l'intérieur d'un discours : il y a des genres épistolaires que l'on peut lire, par exemple, dans le discours médical, dans le discours littéraire ou dans le discours diplomatique. 
sur laquelle il faudrait ici s'attarder est la suivante : puisque les genres sont structurés aussi bien par des normes langagières que socio-cognitives, comment la praxis à laquelle les genres appartiennent conditionne-t-elle la mise en discours des faits de langue ? Comment l'indexation socio-cognitive des genres dans un corpus de textes en français partageant le même genre offre-t-elle la capacité de percevoir une morpho-syntaxe, une énonciation, une argumentation et une rhétorique, par exemple, spécifiques au français, et en même temps différentes de celle de l'anglais ou de l'espagnol ? Qu'apporte à un genre le fait d'appartenir à telle ou telle communauté linguistique ? Peut-il s'en libérer ou pas ? Seule une approche comparatiste, non des textes seuls, mais des genres dans des « communautés ethnolinguistiques » différentes ${ }^{(13)}$, comme lieu de médiation, permettra d'en rendre compte.

\section{4. «Les genres sont des catégorisations dynamiques en variation»}

La variation est un thème central que l'on retrouve dans l'ensemble des travaux qui portent sur la question de la généricité : comment et pourquoi les genres changent-ils ? C'est là une question qui, même après une masse considérable de travaux, demeure à l'ordre du jour pour les linguistes. Nous reprenons ici cette proposition en insistant plus particulièrement sur deux points : le premier est d'ordre conceptuel, le deuxième relève du traitement formel.

Sur la notion de variation, le débat en sciences du langage, il faut l'avouer, est assez confus. Les travaux de F. Gadet (2006) montrent bien comment la confusion a pu s'installer dans le traitement qui lui est consacré en phonologie, en syntaxe ou en sociolinguistique. Pour revenir à $\mathrm{A} \& \mathrm{H}$, leur argumentation établit une différence entre des textes qui tolèrent la variation et d'autres qui sont marqués par le consensus d'une unité dans l'activité langagière. Or encore une fois, on ne nous dit rien sur la manière d'aborder les principes sur lesquels s'appuie le fonctionnement d'un genre, ni sur le statut des variantes à gérer dans le cadre de la nécessaire évolution du genre. Certes, les genres textuels expriment une expérience au monde en évolution constante, puisque la variation est un phénomène naturel propre à toute activité langagière, mais cette proposition ne nous fait pas comprendre ce qui favorise ou non linguistiquement l'occurrence d'une variante. Car le rôle d'une approche dynamique des genres ne consiste pas à raisonner sur les variations langagières ou à établir un savoir sur un genre, mais à baliser les traits spécifiques caractérisant la variation dans tel ou tel genre. Pour être élaborée, une théorie de la variation supposerait que soient définis les paliers de la textualité qui en rendent compte empiriquement. Or A\&H parlent de variation sans qualificatif précis, sans chercher à tenir compte des différents niveaux que celle-ci comporte. On voit le problème que pose au linguiste ce genre de proposition : est-ce que tous les phénomènes sont susceptibles de varier? Il y a donc lieu de se demander si tous les paliers de la textualité manifestent de la variabilité, et si les zones de variation sont repérables et isolables en termes linguistiques. Si nous prenons les genres suivants, " une homélie, un éloge funèbre, un discours politique en présence des membres d'un parti ou un débat politique médiatique, la publicité en général », cités par A\&H pour les opposer aux genres qui sont soumis à des normes strictes, il est difficile de comprendre pourquoi il y a encore aujourd'hui une forte tendance à refuser l'hypothèse de l'hétérogénéité à l'intérieur d'un même genre. L'hétérogé-

(13) C'est dans cette perspective que l'on peut situer, par exemple, les travaux de P. Von Münchow (2007). 
néité est une caractéristique de la structure même des genres, de tous les genres, car la spécificité d'un genre passe aussi par ses variations stylistiques, lexicales, morphosyntaxiques et argumentatives. La notion de genre ne peut être dissociée de celle de «variation ». Tous les textes sont marqués par la variation, et les faits linguistiques que l'on peut qualifier de variants sont au moins aussi nombreux que les invariants. La variation à elle seule ne suffit donc pas à constituer une « proposition ». Car les paliers invariants et variants sont entremêlés dans le tissage du texte. Quelle que soit la manière dont on envisage cette question, il reste à rendre compte de ces constatations. On ne peut pas concevoir autrement le genre que comme l'une des manifestations de la variation dans un discours donné. Mais puisque tout dans un genre ne varie pas, il faut établir une relation entre la part de l'invariant et la part de la variation. Les deux peuvent être des objectifs dignes d'intérêt pour le linguiste. On peut comprendre le désir de parler de variation, mais il faudra catégoriser et nommer les variables et les constantes. Cet aspect de la réflexion reste, aujourd'hui encore, un point de passage obligé pour qui s'intéresse aux problèmes des relations entre genre et variation. Compte tenu de ce qui a été observé plus haut, on pourra se poser les questions suivantes : quels sont les faits de langue du même discours qui peuvent varier ou non d'un genre à un autre ? Quels sont les éléments en variation dans les genres d'un même discours ? Est-ce que, par exemple, c'est la ponctuation qui varie plus et plus librement que la syntaxe dans tous les genres d'un discours contrastés avec ceux d'un autre ? On pourrait aussi s'interroger sur les absents : quels sont les faits de langue dont se prive un genre ? Il est certes difficile de répondre à ces questions sans corpus, mais ce n'est pas une raison pour ne pas s'en donner l'objectif. Il faut continuer à recueillir des corpus génériques pour établir les faits de langue en jeu et leur fonctionnement dans la quête d'une caractérisation multi-sémiotique de l'ensemble des faits relevant de la variation ou de l'invariant qui peuvent se manifester en corpus.

\section{5. « La catégorisation générique se fait par airs de famille, au sein d'un système de genres »}

Un genre textuel en soi n'existe pas, son identité se définit par rapport aux autres genres qui partagent la même pratique que lui, en vertu de certaines ressemblances de «famille». Il est ainsi rattaché à une «forme de vie» dans une pratique sociale historiquement et culturellement déterminée. C'est l'idée globale qu'A\&H soutiennent dans cette proposition qui laisse à penser que le genre demeure tributaire d'un " système de genres ». Or par rapport à leur proposition qui s'appuie essentiellement sur Tomachevski, il convient surtout de souligner le caractère allusif de leur propos. Car parler d' " airs de famille », comme Wittgenstein, cela suppose rendre compte des relations de ressemblance et de différence entre les membres de la même famille. Qu'ont-ils tous de commun pour former un « air de famille » ? Où réside le lien de parenté dans les textes pour parler d'un système de genres? A quelle composante du texte s'attacher pour observer comment l'opérativité des normes se réalise empiriquement? Il y a des apparentements, sans point commun unique, entre les genres de la même famille ; les membres ont plusieurs propriétés en commun, mais il n'y en a aucun qui les possède toutes. A\&H ne disent rien de tout cela, ce qui rend extrêmement délicat la validité de cette proposition. Car parler d' " airs de famille » suppose un espace nettement structuré, porteur d'une mémoire familiale et inscrit dans une dynamique de transmission : paternels d'un côté, maternels de l'autre, ascendants, descendants, alliés ou appartenant à la filiation. Pour être accepté par le 
groupe, le membre doit se soumettre à certaines règles, surtout des règles partagées, des traits (1'air de famille). Mais en même temps la notion d' " airs de famille », il faut le dire, est fluctuante, elle n'a pas de limites précises dans l'espacetemps. Comme les membres d'une famille, les genres sont reliés entre eux d'une multitude de façons différentes et ce sont ces relations multiples qui justifient qu'on leur trouve à tous des « airs de famille». Les liens de parenté font toujours partie de l'univers social des individus. Ces derniers peuvent occuper dans un réseau une place très spécifique, mais la famille est aussi une structure qui se renouvelle à chaque génération. De nombreux facteurs contribuent à modifier constamment la configuration des réseaux familiaux, comme les décès, les naissances, les séparations. Toute la question, pour nous, est de savoir comment les genres sont fondateurs des mécanismes de formation de la norme, comment ils organisent leur réseau de parenté, dont ils portent l'empreinte en respectant un standard usuel de conduites développé à l'échelle des collectifs. Le genre comme système stabilisé n'est pas incompatible avec des déviances que l'usage peut imposer. C'est la question principale que soulève la notion d' " air de famille » qui montre que les membres du système sont régulés, variables et instables malgré leur appartenance à la même activité langagière. Il faudrait, à notre avis, définir ce qu'est un système de genres, comment il fonctionne, comment les normes sont objectivées dans un système de règles, et quel est leur degré d'accessibilité dans une sémiotique des pratiques langagières. Et pour le faire, on a besoin de corpus, et plus précisément de deux types de corpus, comme on le verra à propos du problème de la collecte des données concernant les genres.

\section{6. « La généricité engage tous les niveaux textuels et transtextuels »}

La sixième proposition porte sur les niveaux du texte qui sont affectés par la généricité. Deux composantes du texte, selon A\&H, définissent la généricité : la textualité et la transtextualité. C'est là, sans doute, que réside le point nodal de l'ensemble des problèmes posés par la caractérisation des genres textuels. Les auteurs insistent sur le caractère normatif du genre, en soutenant que « certaines catégories linguistiques peuvent être rendues obligatoires et certaines agrammaticalités rendues acceptables par un genre $»$. Or il faut ici prendre garde au fait que ce n'est pas seulement la catégorie du genre qui est sur ce point concernée, mais toute mise en fonctionnement de la langue par un sujet parlant. Toute activité langagière peut constituer une entrée commode pour penser la tension entre la langue commune aux sujets parlants et les écarts actualisés dans le discours. Ce principe inhérent à l'exercice du langage montre qu'il n'y a pas une pratique unifiée d'un genre. Il ne peut pas exister de genre sans variation et sans régulation, les deux sont nécessaires à son évolution, comme on l'a vu plus haut. Force est pourtant de constater, malgré la justesse de certaines observations, que la discussion sur les forces centrifuges et centripètes, sur les différents niveaux de la textualité et de la transtextualité, sans jamais les confronter à d'autres discours, ne clarifie pas forcément les choses par rapport aux autres propositions. Car finalement, rien ne prouve que ce sont tous les genres ou seulement ceux qui relèvent de la littérature qui sont concernés. Les genres ne peuvent être traités qu'en corpus, et ce que l'on observe dans un corpus uniquement littéraire ne peut pas être généralisé à tous les genres. Pour donner un exemple des limites de la réflexion $\mathrm{A} \& \mathrm{H}$, on constate que plusieurs paliers sont convoqués par les auteurs pour étudier les genres : " sémantique ", « énonciatif» « argumentatif», « compositionnel », « matériel » et « transtex- 
tuel », et qu'ils sont tous sous l'influence directe du genre auquel le texte est rapporté. Rien n'est dit cependant sur l'interaction de ces composantes dans l'analyse de la généricité. Ici le problème essentiel à notre avis, est celui de la corrélation et de l'interaction entre niveaux, et non celui de leur autonomie et indépendance. Nous y reviendrons plus bas, mais disons d'emblée qu'on doit partir d'une corrélation entre plusieurs niveaux du texte si l'on veut caractériser les genres ou la généricité. On ne peut pas réduire le genre à la présence ou l'absence d'un seul phénomène particulier, qu'il soit énonciatif, morpho-syntaxique, argumentatif, compositionnel ou thématique. Car les niveaux qui définissent un genre textuel doivent relever de corrélations entre plusieurs niveaux qui peuvent se succéder ou s'emboîter au sein d'une même interaction, plutôt que de critères appartenant à un seul niveau. C'est précisément là que nous partageons la position de F. Rastier quand il associe genre et interaction : «Un genre étant alors défini comme un mode d'interaction normé entre composantes ». (Rastier et Pincemin 2000 : 96-97).

A quoi on peut ajouter le principe de la fréquence car il faut partir d'une interaction itérative attestée sur corpus entre plusieurs niveaux de la textualité pour caractériser les genres et mettre au jour les niveaux qui contribuent à l'élaboration d'une connexion générique, construite en fonction de contraintes bien particulières imposées par les pratiques langagières. La question des niveaux, c'est aussi le problème de leur compatibilité, dès l'instant où on les conçoit comme un nœud de forces qui se stabilise dans et par le genre. C'est surtout parce que des normes tacitement transmises par les pratiques et dont les locuteurs n'ont pas forcément conscience que des activités langagières régulières se stabilisent puis s'instituent. C'est ainsi que le texte s'ancre dans le genre, à partir des régularités et des écarts qu'il présente à différents niveaux de la textualité. Ainsi se trouvent posées les relations de chaînage multi-sémiotique du genre ou de la généricité. Nous le redisons, notre approche du genre se conforme au postulat suivant : les composantes du texte ne peuvent pas être décrites de façon isolée. Elles ne peuvent pas non plus l'être sans tenir compte de leur fréquence et de leur compatibilité, car rien dans la combinatoire des paliers du texte ne relève du hasard des rencontres fortuites. Le genre est toujours « entre » quelque chose, entre les phrases, entre les paragraphes, entre les différents paliers, entre les textes et entre les différents genres du même discours. C'est la question de la cooccurrence qui nous intéresse ici, et qui a souvent été évoquée en sciences du langage pour des questions lexicales afin d'explorer le rapport fréquentiel dans une paire donnée de lexies co-présentes dans les textes du corpus, et que nous voudrions orienter vers les typologies textuelles. Quels sont les paliers d'un texte qui cooccurrent au sein d'un corpus du même genre ? Par « cooccurrence autoconstituante ${ }^{(14)}$ », nous faisons 1'hypothèse que les textes du même genre partagent un certain nombre de cooccurrences à des niveaux multiples interdépendants, quant à leurs conditions de production et de circulation. Le repérage de ces associations itératives, attestées sur corpus, constitue certainement, quand la taille de l'échantillon est suffisamment grande, un accès privilégié à leur fond générique. Ce sont ces traces d'opérations morphologique, syntaxique, énonciative, argumentative, etc. dont nous n'avons pas toujours conscience, attestées et surtout combinables entre elles dans une série de textes différents qui permettent de faire apparaître les contraintes intrinsèques qui contribuent à l'uniformité des genres. D'un point de vue méthodologique, il s'agit de rappeler que la norme générique est aussi une affaire de fréquence. On cherche ici

(14) $C f$. Ablali (à paraître 2013). 
à montrer que le «plan de l'expression » dans un corpus de textes hétérogènes n'est pas un simple support, il n'est pas extérieur au contenu qu'il est censé véhiculer de manière normalisée.

En clair, il s'agit ici de dire que ces cooccurrences renvoient, implicitement ou explicitement, à une pratique de la langue en suivant la trace d'une tradition générique qui installe un continuum dans les contraintes de l'usage fortement liées à des normes de genre, lesquelles possèdent une systématicité et caractérisent une tradition. En ce point, il est remarquable de souligner que ce sont bien les genres qui transmettent ces cooccurrences et les confirment. Pour le dire autrement, nous emprunterons une citation, parmi de nombreuses autres possibles, aux écrits de E. Coseriu : «La vérité est que les individus parlants possèdent une pleine conscience du système et de ce que l'on appelle les "lois de la langue". Ils ne savent pas seulement ce qu'ils disent mais aussi comment cela se dit (et comment cela ne se dit pas); autrement, ils ne pourraient pas même seulement parler. Il est certain, d'autre part, qu'il ne s'agit pas de "comprendre" l'instrument linguistique (qui est la question du linguiste), mais de savoir l'employer, de savoir maintenir (refaire) la norme et créer en accord avec le système ». (Coseriu 2007).

\section{Perspectives}

Nous sommes presque au terme de notre itinéraire épistémologique et méthodologique. Il ne resterait à mettre en évidence qu'un dernier point, déjà entrevu plus haut : pourquoi avons-nous besoin de la catégorie du genre pour l'analyse des textes et des discours?

Dans la perspective sémiotique qui est la nôtre, deux réponses, et seulement deux à notre avis, sont possibles ici, que nous pouvons résumer en deux points sans aucune intention de réduire ce problème à ces deux constats : le genre comme un espace de variation, et le genre comme une interprétation multi-sémiotique. Par espace de variation, nous voulons dire que le genre ne pourrait laisser certaines formes linguistiques indifférentes. Le sens d'un signe de ponctuation comme les parenthèses dans un texte littéraire n'a pas les mêmes fonctions que celui qu'elles remplissent dans les guides de randonnée ${ }^{(15)}$. L'impératif dans les textes de randonnée n'a pas la même fonction ni les mêmes corrélats que dans une notice médicale. La deuxième relève de la caractérisation multi-sémiotique du genre, caractérisation qui ne peut se faire que sur corpus, et sur « corpus situé », c'est-à-dire sur un corpus construit en tenant compte des frontières des genres et des discours. Et pour insister encore sur cette question des corpus, décidément capitale, nous voudrions faire une distinction entre deux types de corpus pour une caractérisation multi-sémiotique des genres : nous appelons les premiers des corpus « homoprax » : ce sont des textes génériquement hétérogènes qui appartiennent à la même praxis. C'est le cas, par exemple, du discours universitaire que l'on peut étudier sur la base de genres différents comme le rapport de thèse, l'article scientifique et le compte rendu; les seconds sont des corpus «polyprax» : ce sont des textes génériquement homogènes qui relèvent de différentes praxis. C'est le cas, par exemple, du genre épistolaire que l'on peut étudier sur la base d'un corpus issu du discours médical (la lettre du médecin à son collègue), du discours littéraire (les lettre de Cécile à Valmont dans le discours littéraire des Liaisons dangereuses), du

(15) $C f$. Ablali (à paraître 2013). 
discours des " écrits ordinaires 》 (Les lettres d'allocataires adressées à la Caisse d'Allocations Familiales). Il faudra donc accorder davantage de place aux corpus et continuer à recueillir des « corpus situés ". "Les données c'est ce qu'on se donne ", comme dit F. Rastier, et derrière des questions de typologie, il y a un potentiel heuristique qui présente néanmoins l'intérêt d'inciter à des réflexions de linguistique générale et de servir de point d'appui pour d'autres disciplines, comme la syntaxe, la morphologie, la sémantique. Car les études sur les genres sont aujourd' hui le seul observatoire de langue capable d'articuler dans une même configuration des questions linguistiques traditionnellement entreprises dans des espaces disciplinaires distincts.

\section{Bibliographie}

ABLALI, D. (2007) : «Écrire en critique : exploration morpho-syntaxique sur corpus », in Corpus en Lettres et Sciences sociales : des documents numériques à l'interprétation, F. Rastier, M. Ballabriga (dir.). Toulouse, Presses Universitaires de Toulouse Le Mirail, p. 207-214.

— (à paraître 2013) : « La grammaire fonde-t-elle une nouvelle typologie des genres textuels ? La cooccurrence auto-constituante dans les textes de randonnée », in Revue de l'institut de Linguistique Française (revue en ligne). — (2013 b) : «Malaise dans les frontières », Parallèles floues. L'espace théorique du langage, C. Normand et S. Sofia (éds), Bruxelles, Académia, p. 301314.

ADAM, J.-M. (1975) : «Enjeux d'une approche du discours publicitaire : notes pour un travail de recherche $»$, Pratiques n ${ }^{\circ} / 8$, Metz, p. 61-79.

— (1979) : «Ordres du discours : narratif/poétique », Pratiques n²2/23, Metz, p. 105-122.

- (1987) : «Textualité et séquentialité. L'exemple de la description », in Langue française, $\mathrm{N}^{\circ} 74,1987$. p.51-72.

- (1990) : Eléments de Linguistique textuelle, Bruxelles-Liège, Mardaga.

- (1992): Les textes: types et prototypes. Récit, description, argumentation, explication et dialogue, Paris, Éditions Nathan.

— (1998) : «Les genres du discours épistolaire. De la rhétorique à l'analyse pragmatique des pratiques discursives », Siess, Jürgen (éd.) 1998, 37-53.

- (1999) : Linguistique textuelle. Des genres de discours aux textes, Paris, Nathan.

- (2003) : «Entre la phrase et le texte : la période et la séquence comme niveaux intermédiaire de cohésion », in Québec français, $\mathrm{n}^{\circ} 128, \mathrm{p} .51-54$.

— (2004) : «Des genres à la généricité. L'exemple des contes (Perrault et les Grimm)», in Langages $\mathrm{n}^{\circ} 153$, Les genres de la parole, Bouquet, S. (Ed.), Larousse, p. 62-72.

- (2005): "Les sciences de l'établissement des textes et la question de la variation ", in Sciences du texte et analyse de discours, U. Heidmann \& J.-M. Adam (dirs), Genève, Slatkine, p. 69-96. 
- (2006 a) : «Autour du concept de texte. Pour un dialogue des disciplines de l'analyse des données textuelles », JADT 2006, http :/www.cavi.univ-paris3.fr/lexicometrica/jadt/JADT2006-PLENIERE/JADT2006_JMA.pdf.

- $(2006 \mathrm{~b})$ : «Texte, contexte et discours en questions », entretien dans Pratiques $\mathrm{n}^{\circ} 129-130, \mathrm{p} .21-34$.

— (2010) : «L'émergence de la linguistique textuelle en France (1975-2010).

Parcours bibliographique en 100 Titres », Conférence donnée, à l'ENS de Paris, dans le cadre d'une journée CONSCILA (Confrontations en Sciences du Langage), le 28 mai 2010 : «La linguistique textuelle et l'École de Prague: état des lieux».

ADAM, J.-M. \& GOLDENSTEIN, J.-P. (1976) : Linguistique et discours littéraire : théorie et pratique des textes, Paris, Larousse.

ADAM, J.-M. \& HEIDMANN, U. (2004) : « Des genres à la généricité. L'exemple des contes (Perrault et les Grimm) », in Bouquet, S. (dir.), Les genres de la parole, Langages, $\mathrm{n}^{\circ} 153$, Paris, Armand Colin, p. 62-72.

- (2005) : «Sciences du texte en dialogue. Analyse de discours et interdisciplinarité », in Sciences du texte et analyse de discours. Enjeux d'une interdisciplinarité, Genève, Slaktine, p.7-17.

- (2007) : «Six propositions pour l'étude de la généricité », in La Licorne, n79, Le Savoir des Genres, Baroni, R. \& Macé, M. (Eds), p.21-34.

- (2009): Le texte littéraire. Pour une approche interdisciplinaire, LouvainLa-Neuve, Academia Brylant.

Blanchot, M. (1959) : Le livre à venir, Paris, Gallimard.

COSERIU, E. (2007) : «Langue abstraite et langue concrète. La langue comme savoir parler historiquement déterminé. Les trois problèmes du changement linguistique ", Synchronie, diachronie et histoire, (trad. Th. Verjans), http://www.revue-texto.net/Parutions/Livres-E/Coseriu_SDH/Sommaire.html.

DERRIDA, J. (1986) : «La loi du genre », in Parages, Paris, Galilée, p. 249-287.

GADET, F. (2006) :La Variation sociale en français. (Nouvelle édition revue et augmentée). Paris, Ophrys.

HeIDMANN, U. \& ADAM, J.-M., (2010) : Textualité et intertextualité des contes. Perrault, Apulée, La Fontaine, Lhéritier..., Paris, Classiques Garnier.

LOISEAU, S. (2008) : " Corpus, quantification et typologie textuelle », Texto, http ://www.revue-texto.net, octobre 2008, vol. XIII, ${ }^{\circ} 4$.

PEYTARD, J. (1979) : Syntagmes 2, Besançon, PUFC.

RASTIER, F. (1989) : Sens et textualité, Paris, Hachette.

- (2001): Arts et sciences du texte, Paris, PUF.

- (2011) : La masure et le grain, Paris, Champion.

RAstier, F. \& PinCEMIN, B. (1999) : «Des genres à l'intertexte », in Cahiers de praxématique «Sémantique de l'intertexte », $\mathrm{n}^{\circ} 33, \mathrm{p} .83-111$.

PORTILlo SERRANO, V. (2010) : Problématique des genres dans les productions écrites universitaires : cas du résumé scolaire chez des étudiants français et mexicains, Thèse de doctorat soutenue à 1'Université de Franche-Comté en 2010 .

VON MÜNCHOW, P. (2007) : «Les représentations du père dans les guides parentaux français et allemands », in Sellenet, C. (éd.) Les pères en débat. Regards croisés sur la condition paternelle en France et à l'étranger, Ramonville Saint-Agne, Érès, p. 125-136. 